\title{
A Quantitative Analysis of Compromising Emanation from HDMI and Possibility of Information Reconstruction
}

\section{Euibum Lee}

Yonsei University

Dong-hoon Choi

Yonsei University

Taesik Nam

Yonsei University

Jong-gwan Yook ( $\square$ jgyook@yonsei.ac.kr)

Yonsei University

\section{Research Article}

Keywords:

Posted Date: March 3rd, 2022

DOI: https://doi.org/10.21203/rs.3.rs-1364673/v1

License: (c) (i) This work is licensed under a Creative Commons Attribution 4.0 International License. Read Full License 


\title{
A Quantitative Analysis of Compromising Emanation from HDMI and Possibility of Information Reconstruction
}

\author{
Euibum Lee ${ }^{1}$, Dong-hoon Choi $^{1}$, Taesik Nam ${ }^{1}$, and Jong-gwan Yook ${ }^{1, *}$ \\ ${ }^{1}$ Department of Electrical and Electronic Engineering, Yonsei University, Seoul, 03722 Korea, Republic of \\ *jgyook@yonsei.ac.kr
}

\begin{abstract}
This paper presents the electromagnetic interference characteristics of the high-definition multimedia interface (HDMI), a well-known display interface, and quantitatively offers the possibility of information from the leakage in the perspective of radio communication. After analyzing the leakage characteristics, a pseudo-signal model is generated based on the characteristics and behavioral features of the software-defined radio. Subsequently, the model is utilized to investigate the performance of frame-averaging to improve the low signal-to-noise ratio of sensitive information contained in the leakage. In addition, the frame-averaging scheme is quantitatively analyzed under various environmental assumptions. Based on these contributions, the paper offers several indexes that quantitatively present meaningful information in EM leakage. Finally, the actual measurement results are compared with those of the pseudo-model to verify our work.
\end{abstract}

\section{Introduction}

Nowadays, information, treated as a priority asset, constitutes an irreplaceable part of daily human life. Accordingly, related software-security technology is of utmost importance to most users in preventing exposure to extortion threats [1]. However, different types of risks have been reported that could bypass such protection by utilizing radiated emission, which is a physical phenomenon prevalent in the hardware of electronic devices [2]. The technique based on this physical phenomenon is called a side-channel attack, and its purpose is to classify unspecified information such as cryptographic keys [3], display information [4], and other types of eavesdropping. This study intends to focus on the possibility of the information reconstruction from the electromagnetic leakage (EM leakage) from the standardized display interface (SDI) cables, and the related historical background is briefly described. W. van Eck was the first to report the presence of display information in EM leakage in 1985. Moreover, he demonstrated that cathode ray tube (CRT) devices were susceptible to such leakage [5]. Later, Kuhn stated that the EM leakage from digital display systems also contains meaningful information; furthermore, he verified the possibility of reconstruction [6]. Subsequently, Plukovic reported that the information radiated by digital devices is either amplitude- or frequency-modulated within the leakage [7]. Thus, it is reasonable to infer that the information can be partially reconstructed through appropriate sampling and demodulation after signal acquisition.

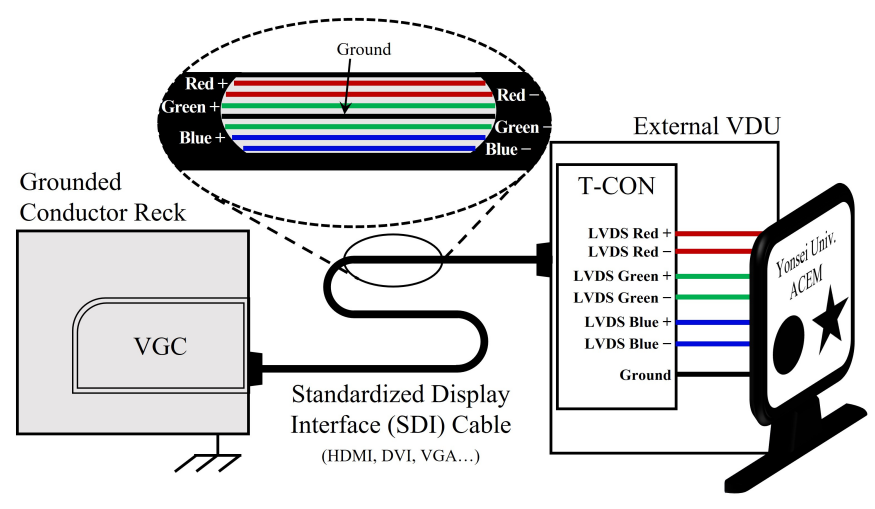

Figure 1. Configuration of generalized video display system 
A video system usually comprises three parts. The first is a video graphic card (VGC) responsible for consistently generating and transmitting hundreds of frames of display information, the second is an SDI cable for video signal transmission, and the third is a video display unit (VDU) that receives the signal and visualizes the information. For better comprehension, a simplified configuration of a video display system is shown in Fig 1.Each component commonly has paths for the electronic signals of display information, and accordingly, it is inevitable that the signal flows on discontinuities entailing EM-leakage bearing the repetitive information. In addition, the SDI and VDU are more likely to experience EM leakage than the VGC, which is typically placed inside the grounded conductor housing. For these reasons, many reports have assumed these two exposed objects as the primary sources of EM leakage and have attempted to acquire meaningful information from them [8]. Specifically, there are analyses regarding the signal generation rules utilized in analog or digital display interfaces [2], [9]; physical principles of EM leakage in the currently-used differential signaling method [10], radiation characteristics, and experimental analysis of leakage signals with an algorithm, to improve the signal-to-noise ratio (SNR) of the compromising emanations [8], [11]. These articles are well-organized based on individual experimental results; however, a majority of them ignore the intuitive relationship between the electronic signal, including the display information, and the EM leakage from the perspective of radio communication. Moreover, when it comes to the frame-averaging scheme, which is commonly utilized to reduce intense noise included in the CE [8], [11], [15], most prior studies have only described the results. A quantitative analysis of the possibility of information reconstruction according to frame-averaging has rarely been discussed.

This study undertakes a thorough investigation of how EM leakage, including leakage of meaningful information, is generated and radiated to the environment. This analysis is based on the signal generation rule of the transition-minimized differential signaling (TMDS) scheme incorporated in the high-definition multimedia interface (HDMI). Subsequently, a pseudo-model of the CE (PCE) is introduced to reflect its spectral characteristics in a specific band. In addition, the pseudomodel reproduces all the operating conditions of the signal acquisition system. Overall, this study determines the principal parameters for signal coherency [12]. Furthermore, it provides a crucial adjustment model to conduct frame-averaging using the implemented PCE.

The implemented pseudo-CE can be combined with various types of noise, such as additive white Gaussian noise (AWGN) or other periodical noise in similar frequency bands that results in the attenuation of the overall signal quality, thereby effectively providing simulation models of realistic acquisition environments. Furthermore, this study introduces a parameter called structural similarity (SSIM) in addition to SNR, which is generally used to quantify background noise. SSIM quantitatively confirms the relationship between the possibility of information reconstruction as a function of SNR. Thus, this paper proposes an index for the possibility of information reconstruction using the frame-averaging process. Finally, for validation, the reconstructed display information from the actual EM leakage of the HDMI is acquired in the EMC chamber and compared with the reconstructions from the implemented noisy PCE, thereby verifying the effectiveness of this pseudo-model analysis.

\section{Analysis of HDMI interface and its signal characteristic}

\section{HDMI Cable}

The HDMI is one of the most popular standard display interfaces. It comprises 19 pin ports and primarily utilizes six pins to transmit the display information: red +, red -, green + , green -, blue + , and blue - in differential signaling. It is known that the leading cause of EM leakage in differential circuits is common-mode noise caused by fabrication errors or timing mismatches [4], [10]. Thus, the signal characteristics of EM leakage are highly relevant to the signaling in HDMI, and its investigation gives excellent insight into the leakage of sensitive information.

\section{Characteristics of the HDMI interface signal}

The VGC generates display information according to the standard preset defined by the 'Video Electronics Standards Association (VESA)' [14]. The preset includes two specific divisions: the display region and blank region. So, for example, given the display in a VDU of the alphabet 'A' as shown in Fig. 2-(a), the signals in the HDMI cable include the pixel information of the display region as well as the pixels in the blank region (PBR). Such an empty area defined by the VESA is similar to most other SDIs.

As shown in Fig. 2-(a), $x_{t}$ and $y_{t}$ are the total numbers of pixels in the horizontal and vertical directions, respectively, and $x_{d}$ and $y_{d}$ denote the partial ranges where visible pixels exist. Therefore, if each pixel constituting the display information of Fig. 2-(a) is assumed as a personal space, as shown in Fig. 2-(b), the transmission of information can be represented by filling the respective area with a specific symbol indicating the value of the pixel. In the case of HDMI, an encoding technique called transition-minimized differential signaling (TMDS) provides a particular value for each space. It comprises a 10-bit sequence for the color of a pixel during a time interval $T_{p}$. For better understanding, take the $5^{\text {th }}$ row of display information shown in Fig. 2-(a) as an example; it consists of a consecutively alternating sequence: a white bit sequence lasting for $6 T_{p}$, a black bit sequence lasting for $6 T_{p}$, another white bit sequence lasting for $7 T_{p}$, and finally, the bit sequence representing the PBR lasting for $3 T_{p}$. Therefore, if we reveal the bit sequence of the respective $\mathrm{R}, \mathrm{G}$, and $\mathrm{B}$ values in each space, the frequency characteristic 


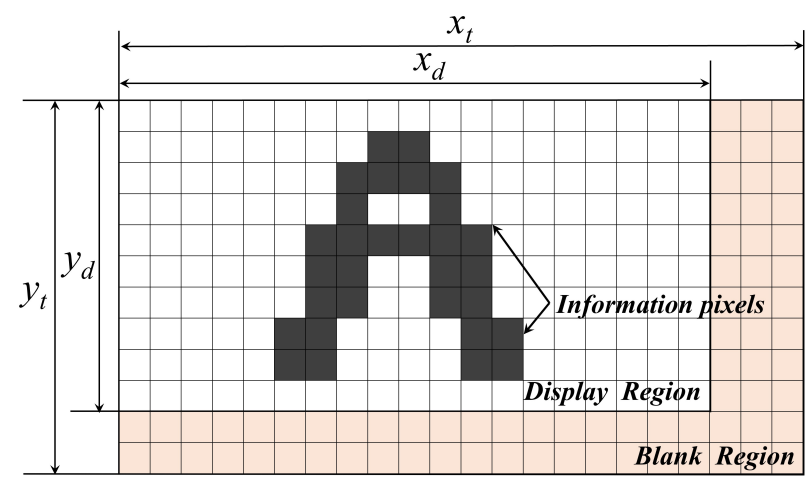

(a)

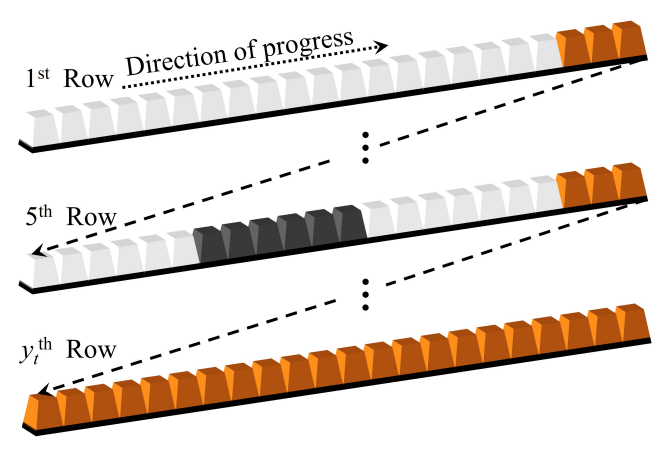

(b)

Figure 2. (a) Configuration and (b) conceptual arrangement of the display information

of the current signal flow is available when the pixel's color is given. The specific TMDS bit sequence generation principle is well described in [4]; in this study, the bit sequences of the black and white pixels are described in addition to the PBR in Fig. 3 as an example. These are the most elementary pixel compositions constituting a document.

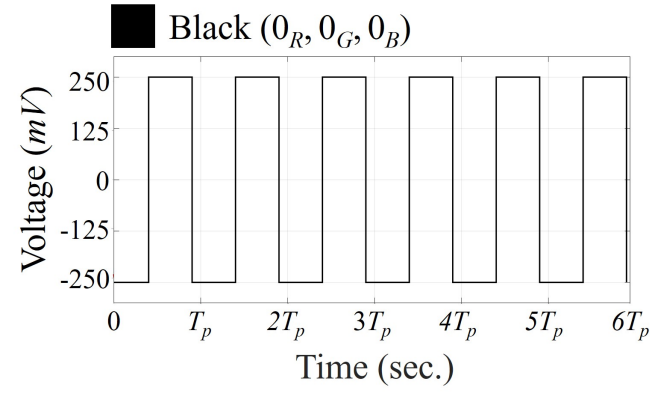

(a)

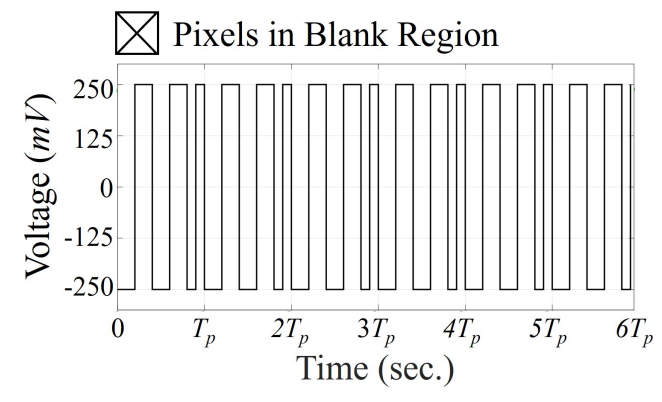

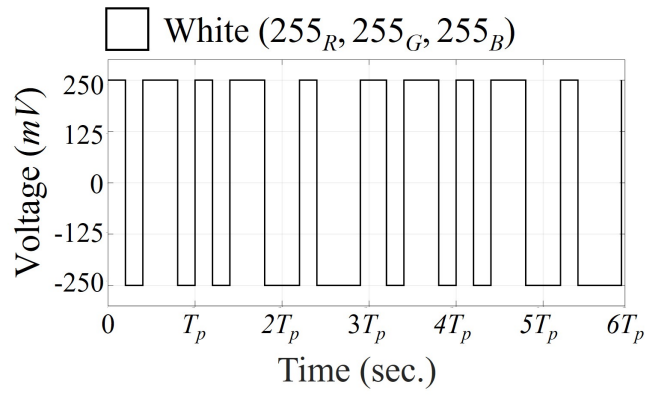

(b)

(c)

Figure 3. Bit sequence of (a) black, (b) white color and (c) pixel in blank region

As shown in Fig. 3-(a), it is evident that the constant bit sequence repeats at $T_{p}$ intervals for black pixels. In the case of white pixels, there are two types of bit sequences repeating twice and once during a total of $3 T_{p}$ intervals, as shown in Fig. 3-(b). Regarding the PBR, there exist four types of bit sequences in reality [4], but for the sake of convenience, this study focuses on a specific bit sequence that generally occupies more than $90 \%$ of its pixel space. As such, because the bit sequence representing the pixel of a particular color continuously changes with time, it is evident that the signal characteristics of EM leakage emitted from HDMI also change instantaneously. Therefore, in a specific duration where the same bit sequence continuously appears, the EM leakage can be considered a Fourier series. This series can be assumed to be composed of harmonic components associated with $f_{p}$, which is the reciprocal of $T_{p}$. Interestingly, the white pixel, the bit sequences of which are consecutively 
repeated every $3 T_{p}$, presents a harmonic component with $f_{p} / 3$ separation. For better comprehension, a spectrogram of the $5^{\text {th }}$ row of Fig. 2(a) is illustrated in Fig. 4.

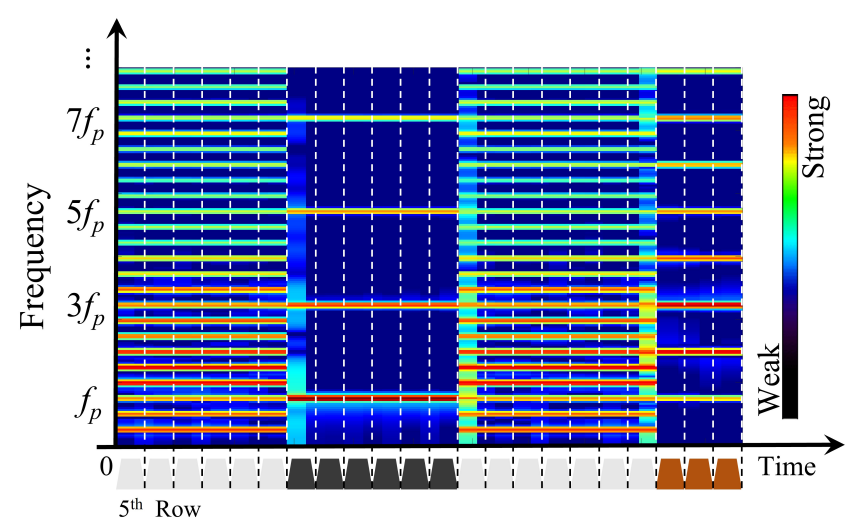

Figure 4. Spectrogram of the $5^{\text {th }}$ row presented in Fig. 2-(a)

Figure 4 shows that the inherent $f_{p}$-related harmonic components for three different pixels are distinctly visible during their time period. Moreover, it is clear that such analytical approaches can be applied similarly to all other colors represented by pixel values. In addition, because the HDMI repeatedly transmits the number of pixels constituting a frame $\left(x_{t} \cdot y_{t}\right)$ through serial communication following the direction of progress, as illustrated in Fig. 2-(b), the spectrum shown in Fig. 5 can be obtained. Note that the length of one frame, $T_{f r}$, is given as $x_{t} \cdot y_{t} \cdot T_{p}$.

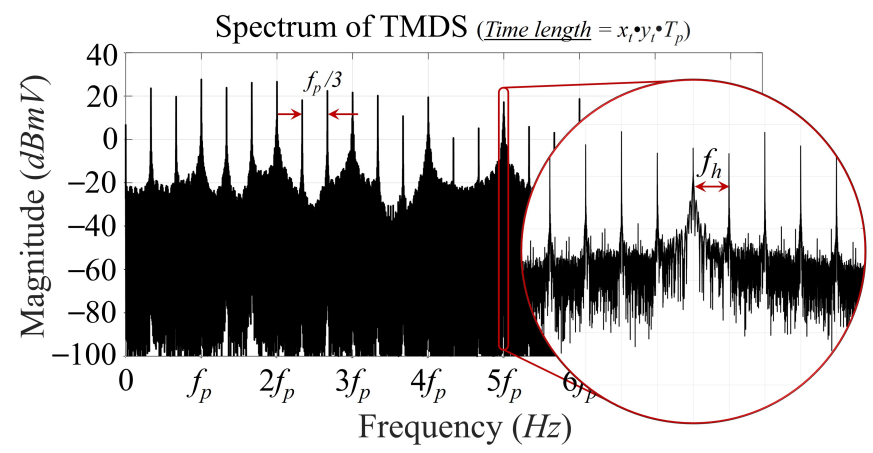

Figure 5. Frequency spectrum during $T_{f r}$

It is noteworthy that by stretching a specific harmonic band, the center frequency of which $f_{c}$ is associated with $f_{p}$, various distinct frequency components are observed, as shown in the circle of Fig. 5. These are observed at an interval of the horizontal synchronization frequency, $f_{h}$, which is the reciprocal of the horizontal sync periodicity $\left(T_{h}=x_{t} \cdot T_{p}\right)$. This phenomenon occurs because the pixels are arranged with a vertically identical color as well as the PBR in the range of $y_{d}$ rows. Interestingly, such spectral characteristics are similar to those of the baseband signal modulated at multiple carrier frequencies. Because the radiation of the EM leakage starts with a rapid transition of current flow in the circuit, the spectral characteristics of the HDMI signal are extremely useful in analyzing the corresponding EM leakage.

\section{Pseudo-models of the CE and frame-average scheme}

The previous section concluded that the display configuration along the pixel arrangement determines the baseband characteristics. Moreover, the shape and combination of the TMDS bit sequence assigned to each pixel determines the carrier frequency, which modulates the baseband signal. This section proposes a PCE model through a joint analysis of the operational characteristics of a real-time software-defined radio (SDR) used for signal acquisition. The model offers a quantitative analysis of the frame-averaging technique used for display information reconstruction. 


\section{Modeling of the Pseudo-CE}

Most display devices express the values of each pixel with respective 8-bits for red, green, and blue. In addition, in the case of the HDMI, R, G, and B values are converted into TMDS codes and transmitted in parallel. Unfortunately, because of the excessive number of cases (16,777,216 for 24-bit), when every color of the pixel is examined, this study assumes only five achromatic colors in which the R, G, and $\mathrm{B}$ values of the pixels in the display region are identical to each other. The five colors that were selected are: black $\left\{0_{R}, 0_{G}, 0_{B}\right\}$, dark gray $\left\{63_{R}, 63_{G}, 63_{B}\right\}$, gray $\left\{127_{R}, 127_{G}, 127_{B}\right\}$, light gray $\left\{191_{R}, 191_{G}, 191_{B}\right\}$, and white $\left\{255_{R}, 255_{G}, 255_{B}\right\}$. First, to analyze the radiation emission caused by the signal flow in the HDMI, a near-field $\mathrm{H}$-field probe and a high-speed digital oscilloscope were utilized, as illustrated in Fig. 6.

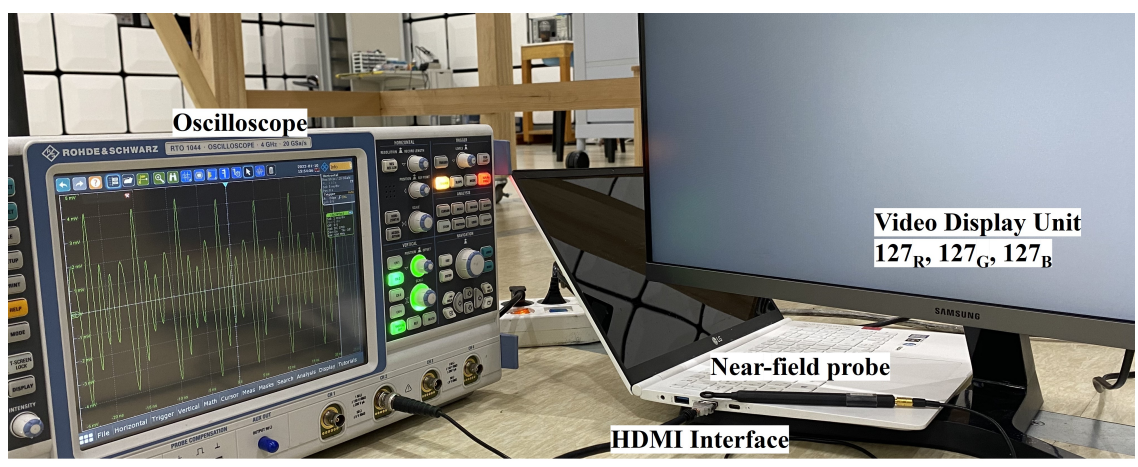

Figure 6. Measurement of EM-leakage according to the pixel's color

The acquisition time of the leakage was more than $100 T_{p}$. Fig. 7 presents the spectrum and corresponding waveforms of the six types of pixels (five achromatic colors + PBR), checking their harmonic configuration according to the TMDS bit sequences assigned to each pixel.
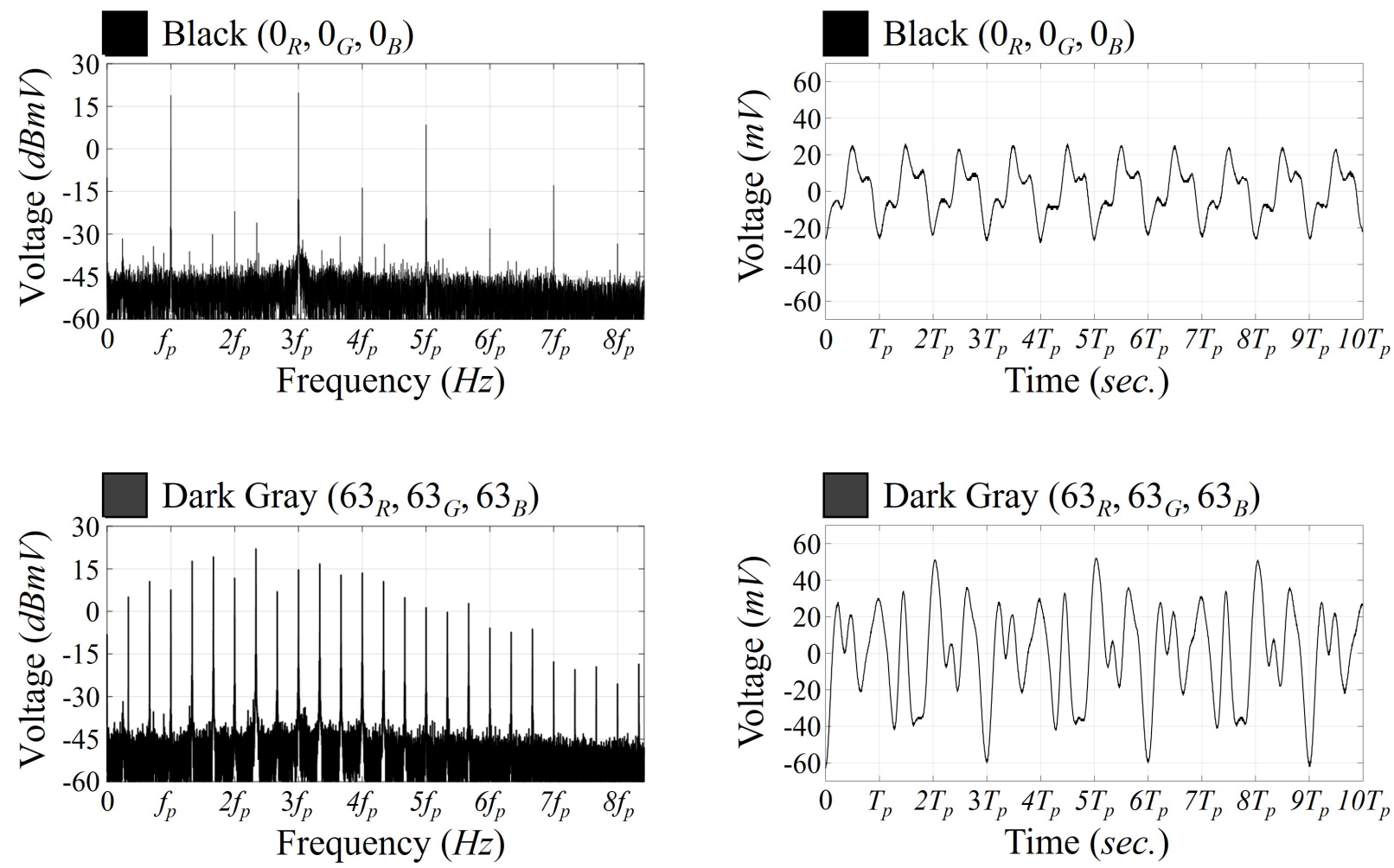

Figure 7. Spectrum and corresponding waveforms of the six pixels 

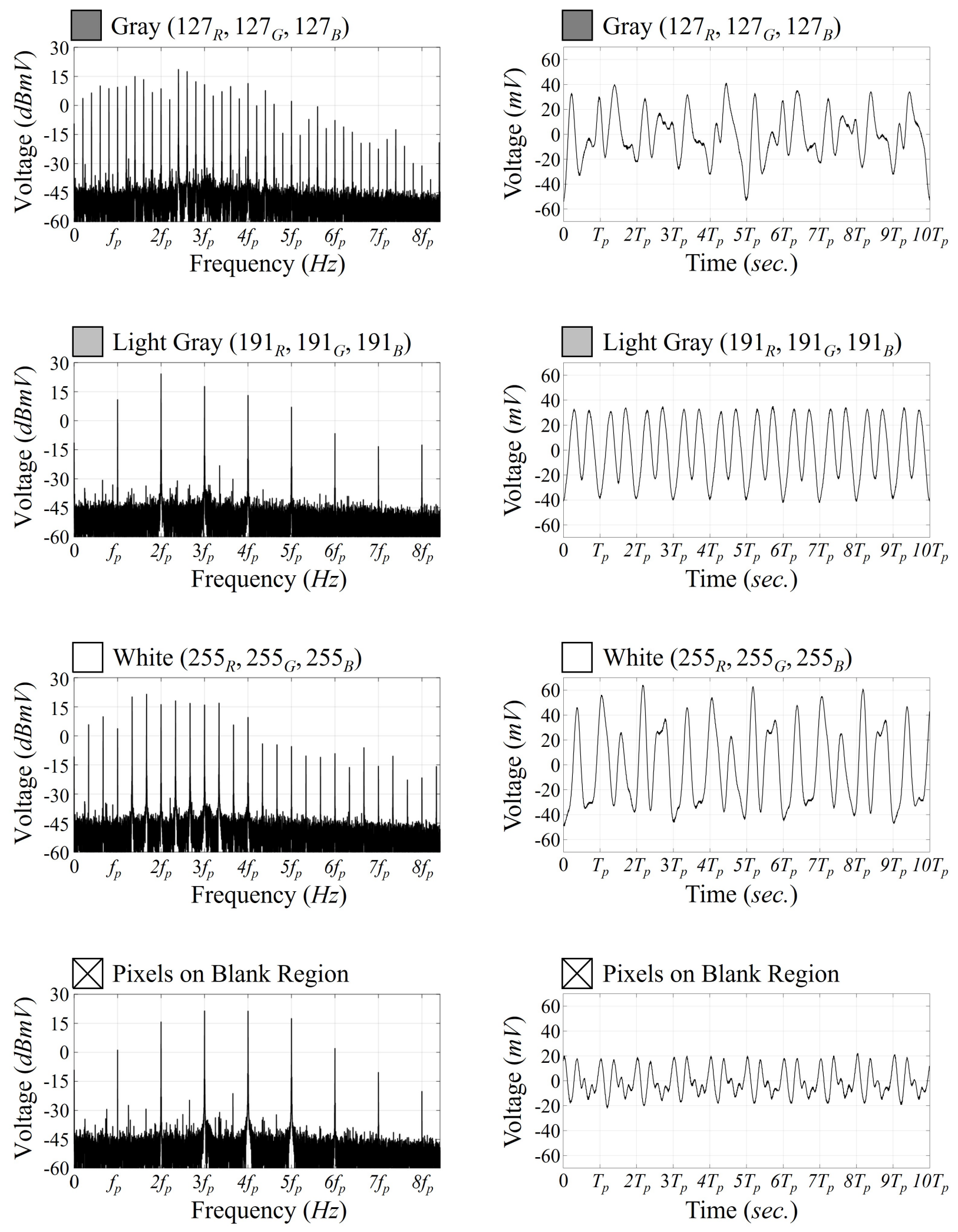

Figure 7. Spectrum and corresponding waveforms of the six pixels (Cont.) 
As is clearly shown in Fig. 7, the dark gray pixel manifests an alternatively repeating EM leakage waveform with a $3 T_{p}$ period and $f_{p} / 3$ interval in its harmonic spectrum, similar to the white pixels. In addition, in the case of the gray pixel, the total period of which is $5 T_{p}$, also presents an $f_{p} / 5$ interval. Consequently, the harmonic configuration of the color of the respective pixel can be defined as (1).

$$
\begin{aligned}
\sigma_{\text {black }}(t) & =\sum_{a=1}^{A} w_{\text {black }}(a) \cdot \cos \left\{a f_{p} t+\phi_{\text {black }}(a)\right\} \\
s_{\text {d.gray }}(t) & =\sum_{a=1}^{A} \sum_{b=0}^{2} w_{\text {d.gray }}(a, b) \cdot \cos \left\{\left(a+\frac{b}{3}\right) f_{p} t+\phi_{\text {d.gray }}(a, b)\right\} \\
s_{\text {gray }}(t) & =\sum_{a=1}^{A} \sum_{c=0}^{4} w_{\text {gray }}(a, c) \cdot \cos \left\{\left(a+\frac{c}{5}\right) f_{p} t+\phi_{\text {gray }}(a, c)\right\} \\
s_{\text {l.gray }}(t)= & \sum_{a=1}^{A} \sum_{b=0}^{2} w_{\text {l.gray }}(a, b) \cdot \cos \left\{\left(a+\frac{b}{3}\right) f_{p} t+\phi_{\text {l.gray }}(a, b)\right\} \\
s_{\text {white }}(t)= & \sum_{a=1}^{A} \sum_{b=0}^{2} w_{\text {white }}(a, b) \cdot \cos \left\{\left(a+\frac{b}{3}\right) f_{p} t+\phi_{\text {white }}(a, b)\right\} \\
s_{P B R}(t)= & \sum_{a=1}^{A} w_{P B R}(a) \cdot \cos \left\{a f_{p} t+\phi_{P B R}(a)\right\} .
\end{aligned}
$$

In (1), $A$ is a positive integer, and $s_{\text {color }}$ are the noise-free time-domain signals, as shown in Fig. 7, according to its subscript color. The EM leakage appears in a broad frequency band; thus, if a particular band including a specific harmonic component of the respective pixel can be collected among the multiple configurations, it is possible to distinguish the difference in pixel values using various demodulation methods [15]. Here, for the band-limited signal collection, a real-time SDR comprising down-conversion, filters, and a continuous signal sampler can be utilized, as shown in Fig. 8, [15], [16].

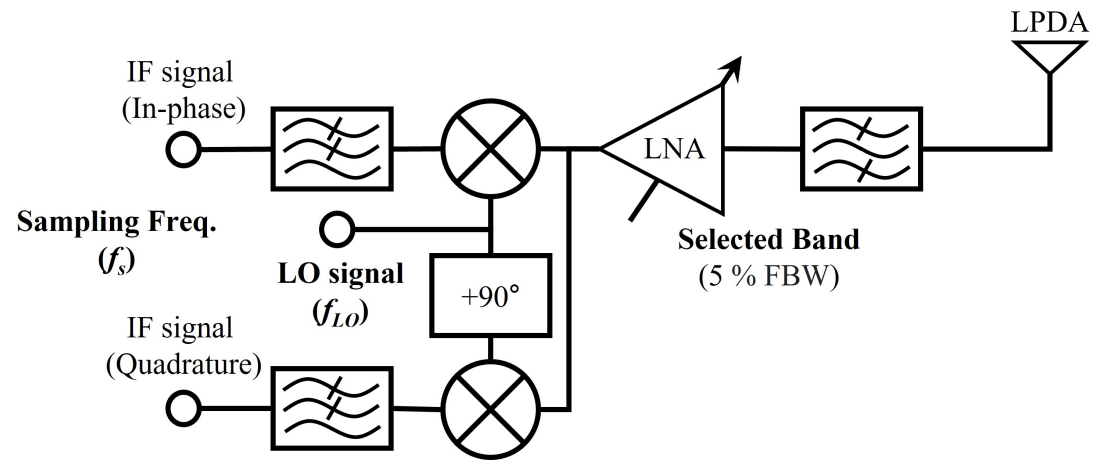

Figure 8. Configuration of the real-time SDR

In the SDR, there exists a trade-off between the SNR and bandwidth of the acquired signal. Consequently, it is crucial to select an appropriate bandwidth. In this study, it was assumed that the bandwidth of the radio frequency (RF) front-end was approximately $5 \%$ of the fractional bandwidth (FBW) at a specific center frequency $\left(f_{c}\right)$. In addition, the bandwidth is set so as not to exceed $f_{p} / 3$. For instance, this study deals with the process of generating PCE after collecting a specific band, the center frequency of which is $(3+2 / 3) f_{p}$, using the SDR under the previous bandwidth assumption. Note that the frequency is selected because it is the middle frequency among the effective frequency ranges. Assuming that the operation frequency of the down-conversion mixer in the SDR $\left(f_{L O}\right)$ shifts the selected band, the center of which is $f_{c}$, to the origin, as shown in (2), the spectrum of the resultant pixel is presented in Fig. 9.

$$
S_{\text {color filtered }}(f)=S_{\text {color }} \cdot H_{B P F} \text { of } 5 \% * \delta\left(f-f_{L O}\right)
$$

In (2), $S_{\text {color }}(f)$ is a spectrum of $s_{\text {color }}(t)$ in (1). As shown in Fig. 9, there are three types of pixels, dark gray, gray, and white that exist on the selected band. In addition, the relative magnitude can be derived using Parseval's theorem, as in (3). The 


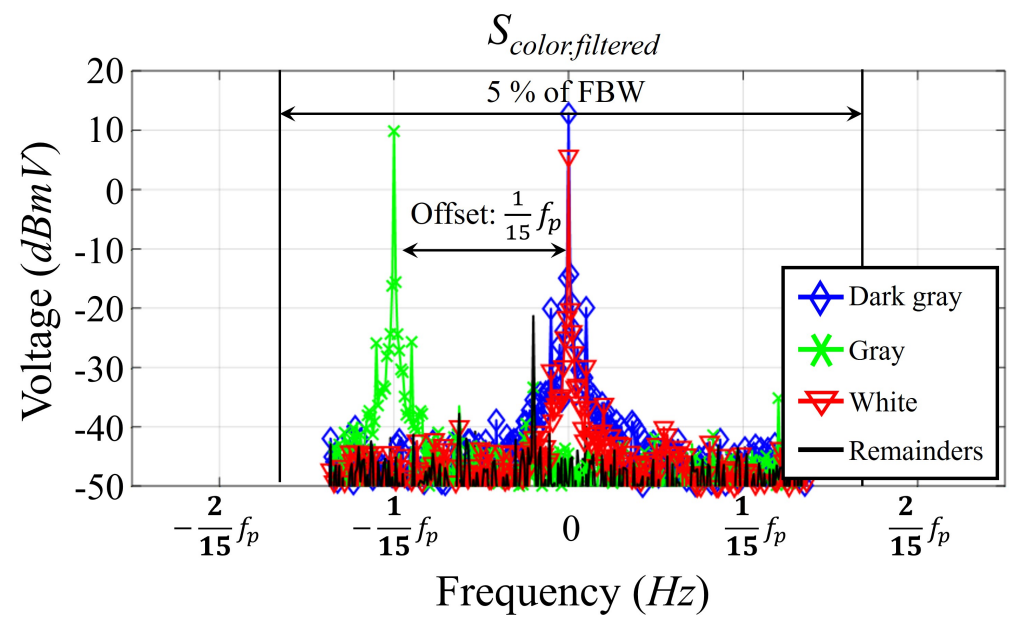

Figure 9. Spectrum of the existing Pixel in the limited band of $\left(3+\frac{2}{3}\right) f_{p}$

verification is conducted on the $(3+2 / 3) f_{p}$ component with an $\mathrm{RF}$ front-end having a bandwidth of $5 \%$.

$$
V_{\text {color }}=\sqrt{\int_{-\infty}^{\infty} S_{\text {color.filtered }}^{2}(f) d f}
$$

TABLE I: $V_{\text {color }}$ on $(3+2 / 3) f_{p}$

\begin{tabular}{|c|c|c|}
\hline$V_{\text {Black }}$ & $V_{\text {D.gray }}$ & $V_{\text {Gray }}$ \\
\hline 0 & 255 & 180 \\
\hline$V_{\text {L.gray }}$ & $V_{\text {White }}$ & $V_{P B R}$ \\
\hline 0 & 110 & 0 \\
\hline
\end{tabular}

Note that the values were normalized within an 8-bit expression. To utilize the $V_{\text {color }}$ results in the configuration of the baseband, two types of display information containing only the five types of achromatic color pixels are adopted and subsequently transformed into the pixel magnitude configuration according to Table I, as shown in Fig. 10.

Now, the baseband configuration for the PCE is achieved by allocating the converted display information to the Display region, as shown in Fig. 2-(a), and filling the remaining blank region with the PBR magnitude derived in the previously selected $(3+2 / 3) f_{p}$ band. Furthermore, as shown in Fig. 9, the gray pixel, which includes $(1 / 15) f_{p}$ of the frequency offset from the origin, should be considered. In this study, instead of using a constant value of 180 , the sample of gray utilizes a complex value of $180 \cdot e^{j\left(2 \pi \frac{1}{15} n+\phi_{s}\right)}$. Here, $n$ is the order of samples in a discrete signal, while $\phi_{s}$ is a term used to initialize the phase when a bundle of consecutive gray pixels re-emerges. Given that the complete configuration of the baseband signal is arranged in a serial format according to the direction of progress described in Fig. 2-(b), a discrete signal of $S_{\text {base }}[n]$ comprises $x_{t} \cdot y_{t}$ number of samples, constituting one frame. In addition, VDS continuously repeats the baseband signal as follows;

$$
S_{\text {base }}[n]=S_{\text {base }}\left[n-x_{t} y_{t}\right] \text {. }
$$

Now, the re-sampling of (4) with $T_{p}$ can be written as presented in (5);

$$
S_{\text {base }}(t)=\sum_{n=-\infty}^{\infty} S_{\text {base }}\left(n T_{p}\right) \cdot \operatorname{sinc}\left(\frac{t-n T_{p}}{T_{p}}\right),
$$

where, $S_{\text {base }}(t)$ is the original signal at the center frequency of $f_{c}$, and the down-conversion frequency, $f_{L O}$, is used in SDR. Now, the received signal right after the RF front-end can be modeled as;

$$
S_{\text {received }}(t)=S_{\text {base }}(t) \cdot e^{j 2 \pi\left(f_{c}-f_{L O}\right) t}
$$




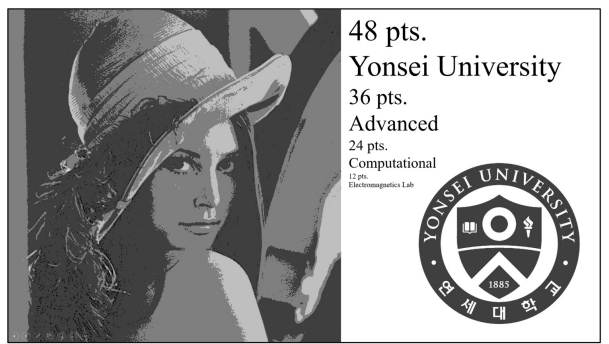

Original achromatic

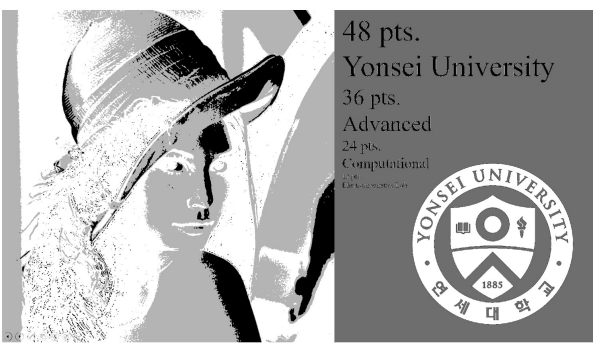

Converted into $(3+2 / 3) f_{p}$

(a)

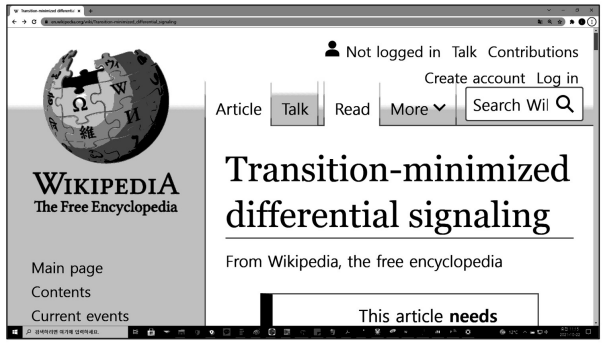

Original achromatic

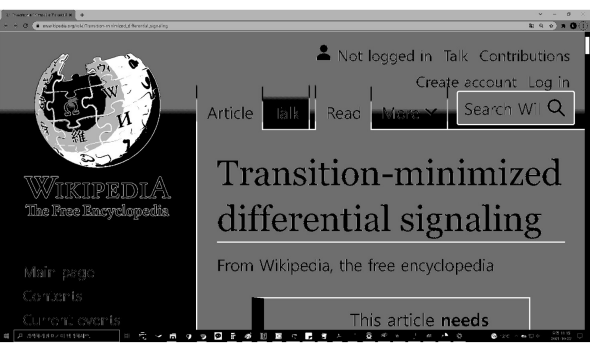

Converted into $(3+2 / 3) f_{p}$

(b)

Figure 10. 5-color based achromatic display information

Figure 11 shows a comparison between the spectrum of the pseudo- $S_{\text {received }}$ and the actual- $S_{\text {received }}$ observed using the oscilloscope. The time length of the Fourier transform is defined as $T_{f r}$.

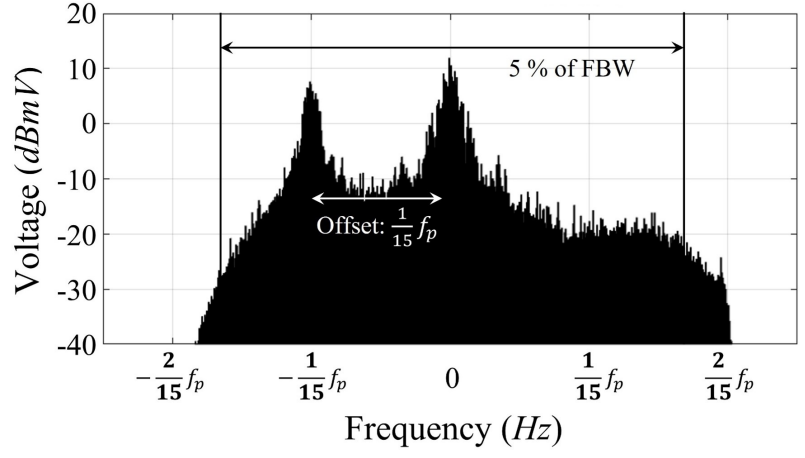

(a)

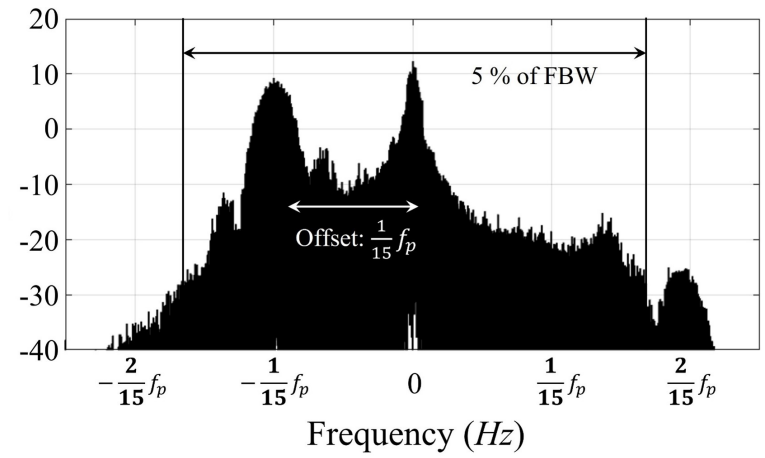

(b)

Figure 11. Spectrum of (a) pseudo $S_{\text {received }}(t)$ and (b) actual $S_{\text {received }}(t)$

As shown in Fig. 11, the spectrum characteristics of the pseudo-signal and the actual-signal from $(3+2 / 3) f_{p}$ are quite similar. Taking this into consideration, we now discuss the behavioral properties of the sampling and windowing process for the real-time SDR using the implemented pseudo-signal. It must be noted that a tiny tolerable range exists for every electronic device; consequently, the VDS cannot update the display information while perfectly following $T_{f r}$, defined by VESA. This is illustrated in equation (7) in which the correlation between the accurate sampling rate of $\operatorname{SDR}\left(f_{s}\right)$ and $f_{p}$, including some errors, is expressed using $\Delta$. In addition, the natural number $P$ ensures at least one sample collection for one-pixel information.

$$
P \cdot f_{p}=\Delta \cdot f_{s} \quad(P \in \mathbb{N})
$$

The required sampling time for an approximate single frame $\left(T_{\text {window }}\right)$, as many as the number of pixels $\left(x_{t} \cdot y_{t}\right)$, can be expressed 
as;

$$
T_{\text {window }}=\frac{P}{f_{s}} \cdot x_{t} \cdot y_{t}
$$

Here, by considering (7) and (8) simultaneously, a time difference can be observed between $T_{\text {window }}$ and $T_{f r}$ owing to $\Delta$ as;

$$
T_{\text {window }}=\Delta \cdot T_{f r} .
$$

Additionally, because of the unknown error parameter, $\Delta$, the previously defined $f_{L O}$ can be expressed as;

$$
f_{L O}=\Delta \cdot f_{c} .
$$

This error prevents the down-conversion from cancelling out the exponential term in (6), eventually causing a carrier frequency offset. Finally, by considering the sampling characteristics of the ADC in the real-time SDR, a pseudo-CE with a length of $T_{\text {window }}$ can be obtained as;

$$
S_{f r . k}(t)=\sum_{n=0}^{N-1} s_{b a s e}\left(t-\tau_{k}\right) \cdot e^{j 2 \pi\left(f_{c}-f_{L O}\right)\left(t-\tau_{k}\right)} \cdot w(t) \cdot \delta\left(t-\tau_{k}-\frac{n}{f_{s}}\right) .
$$

(but, $\left.k=0,1,2, \ldots, K-1, N=x_{t}, y_{t}\right)$

Here, $\tau_{k}$ is $k \cdot T_{\text {window }}$ and $w(t)$ is a window function whose time length is $T_{\text {window }}$ from the origin. $S_{f r . k}$ shows that the VDS clock error $(\Delta)$ and carrier frequency offset error $\left(f_{\text {carr }}-f_{L O}\right)$ are accumulated in proportion to $k$. Therefore, in order for the sampled signal $s_{f r k}[n]$ to satisfy the coherency for every $k$, time error correction as much as $\tau_{k}$ and phase error correction as much as $\tau_{k}\left(f_{c a r r}-f_{L O}\right)$ must be conducted. The compensation reflecting these can be expressed as;

$$
S_{a d j . k}[n]=S_{f r . k}[n] * \delta[n+k \Delta] \cdot e^{-j 2 \pi k \Delta\left(f_{c}-f_{L O}\right)} .
$$

To obtain the parameters $k \Delta$ and $\left(f_{c a r r}-f_{L O}\right)$, auto-correlation [11] and carrier frequency offset estimation can be utilized [13].

\section{Quantitative analysis of information reconstruction possibility}

This section describes the possibility of information reconstruction by implementing the PCE model. The CE includes sensitive information in various modulated forms, and several preceding studies have described that the most effective demodulation technique is amplitude demodulation, as presented in (13) [4], [5], [8], [11], [17], [18];

$$
u_{A M}[n]=\sqrt{S_{f r k k}[n] \cdot S_{f r . k}^{*}[n]} .
$$

After applying the demodulation for $S_{f r \cdot k}$, the reconfiguration of the display information is possible in accordance with the display configuration shown in Fig. 2-(a). However, in a realistic environment, the CE is combined with noise, the distribution of which follows the Gaussian. Hence, $S_{\text {noisy }}$ that comprises $S_{f r}$, and $N_{A W G N}$ is newly introduced in the analysis as in (14) and its SNR can be defined as in (15).

$$
\begin{aligned}
& S_{\text {noisy }}=S_{f r}+N_{A W G N} . \\
& S N R[\mathrm{~dB}]=20 \cdot \log _{10} \frac{S_{f r(R M S)}}{N_{A W G N(R M S)}} .
\end{aligned}
$$

For instance, there are two kinds of $S_{\text {noisy }}$; one of which, $S_{\text {noisy } 1}$, includes the conditions; $P=1, f_{c}=(3+2 / 3) f_{p}, \Delta=100.0001 \%$, $\left(f_{c}-f_{L O}\right) / f_{L O}=0.000091 \%$ and SNR $=-6.01 \mathrm{~dB}$ based on Fig. 9-(a) as $S_{\text {base }}$, while the other, $S_{\text {noisy2 }}$, has the conditions; $P=$ $1, f_{c}=(3+2 / 3) f_{p}, \Delta=99.99989 \%,\left(f_{c}-f_{L O}\right) / f_{L O}=-0.00009 \%$ and SNR $=-5.99 \mathrm{~dB}$ based on Fig. 9 -(b) as $S_{\text {base }}$. Because $P$ is 1 for both cases, the number of samples for a single $k$ is $x_{t} \cdot y_{t}$. Accordingly, the reconfiguration can be achieved by arranging $u_{A M}\left[S_{n o i s y}\right]$ in a two-dimensional format, as shown in Fig. 2-(a), and the results for different $k$ are illustrated in Fig. 12.

As shown in Fig. 12, most of the information included in the PCE is likely not achievable with only a single $k$ of $S_{f r}$ owing to the considerable amount of additive noise. Therefore, to define the possibility of the reconstructed display information from 


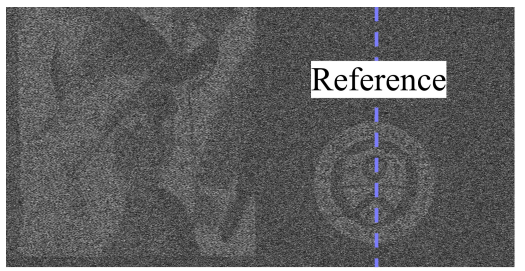

$k=0$

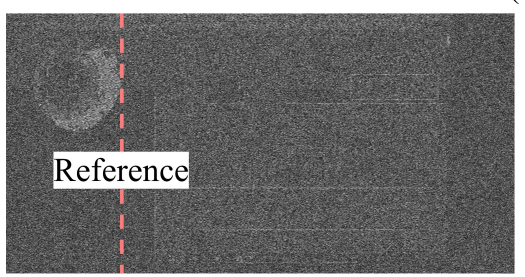

$k=0$

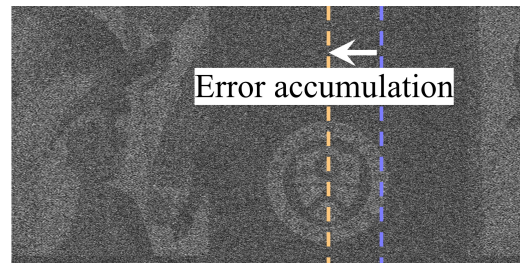

$k=100$

(a)

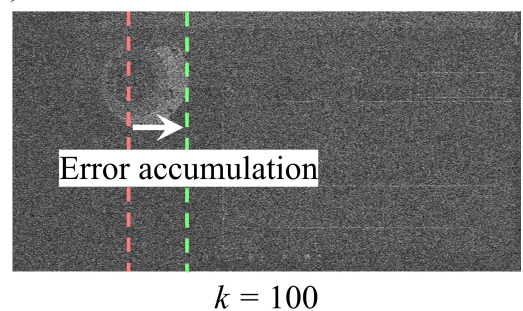

(b)

Figure 12. Reconstructed display information of the (a) $S_{\text {noisy1 }}$ and (b) $S_{\text {noisy2 }}$ with different $k$

such a noisy PCE, we utilized a different index called structural similarity (SSIM), which is defined as an objective method to quantify the visual difference between a distorted and reference image [19];

$$
\operatorname{SSIM}_{S_{1}, S_{2}}=\frac{\left(2 \mu_{S_{1}} \mu_{S_{2}}+c_{1}\right)\left(2 \sigma_{S_{1} S_{2}}+c_{2}\right)}{\left(\mu_{S_{2}}^{2}+\mu_{S_{2}}^{2}+c_{1}\right)\left(\sigma_{S_{1}}^{2} \sigma_{S_{2}}^{2}+c_{2}\right)} .
$$

In (16), $\mu_{S_{1}}, \mu_{S_{2}}$, and $\sigma_{S_{1}}, \sigma_{S_{2}}$ are the average and variance of $S_{1}$ and $S_{2}$, respectively, and $\sigma_{S_{1} S_{2}}$ is the covariance between $S_{1}$ and $S_{2}$. In addition, $c_{1}$ is set as $(0.01 \cdot 255)^{2}$ and $c_{2}$ is set as $(0.03 \cdot 255)^{2}$ in this work. Given that $S_{1}$ is $u_{A M}\left[S_{f r k}\right]$ as the reference signal and $S_{2}$ is $u_{A M}\left[S_{\text {noisy.k }}\right]$ as the distorted signal, the SSIM variation as a function of SNR is obtained, as shown in Fig. 13.

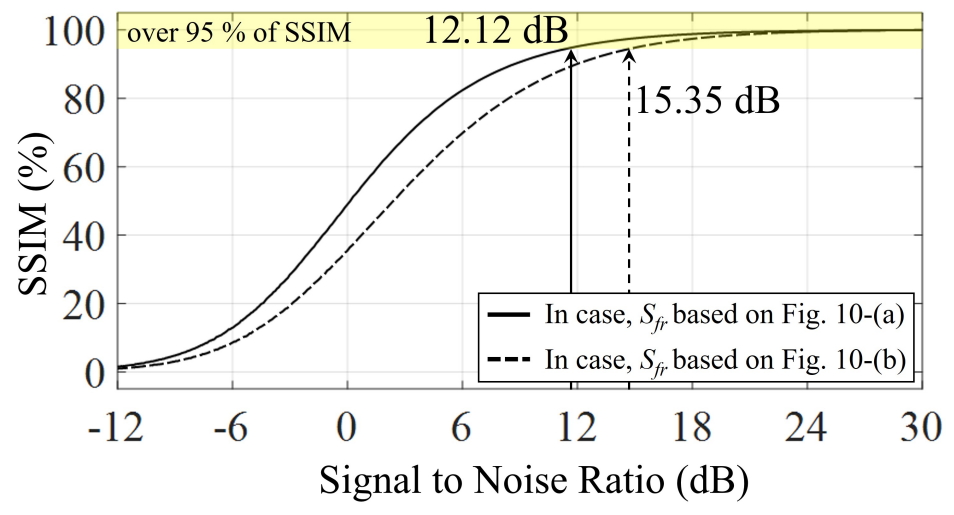

Figure 13. SSIM variation as a function of SNR

The reconstructed results shown in Fig. 12, the SNR of which is approximately $-6 \mathrm{~dB}$, hardly provide any useful information. It measures under $12.5 \%$ on the SSIM. Thus, to obtain meaningful data from the CE, the SNR of $S_{\text {noisy }}$ must be improved to a certain level. For this purpose, this study introduces the frame-averaging scheme described in (17). However, because of the error accumulations shown in Fig. 12, the reconstructed information contains different compositions according to $k$. Hence, it conducts adjustments using (12) between the $S_{f r . k}$ with different $k$ in advance.

$$
S_{f a}[K]=\frac{1}{K} \sum_{k=0}^{K-1}\left(S_{a d j . k}+N_{A W G N}\right)=S_{f r .0}+\frac{N_{A W G N}}{K} .
$$


In (17), it is evident that the white noise decreases according to the number of averaged frames $(K)$. The SNR variation according to the number of averaged frames $K$ can be derived as (18), and a related graph is presented in Fig. 14.

$$
S N R[K][d B]=20 \cdot \log _{10} \frac{\left(S_{f r .0}\right)_{R M S}}{\left(\frac{N_{A W G N}}{K}\right)_{R M S}}=20 \cdot\left\{\log _{10} \frac{\left(S_{f r .0}\right)_{R M S}}{\left(N_{A W G N}\right)_{R M S}}+\log _{10} \sqrt{K}\right\}
$$

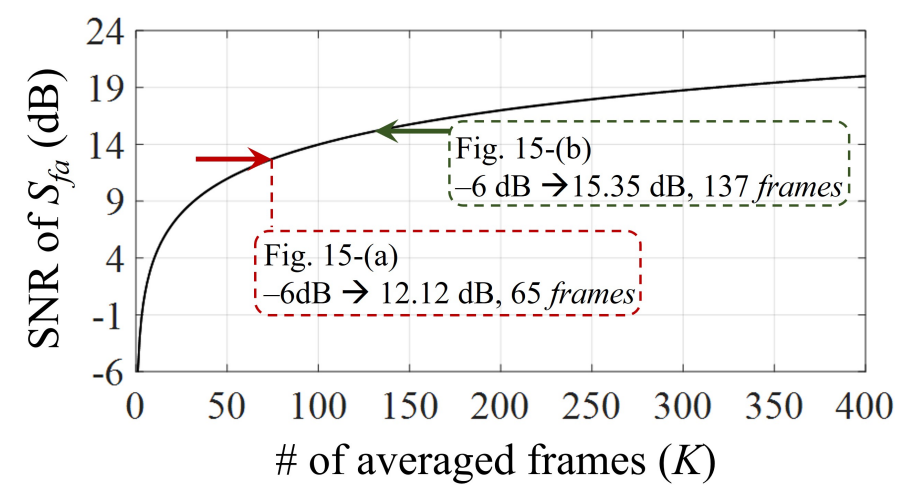

Figure 14. Increment of SNR as a function of averaged frame number $(K)$

Considering a minimum value of $95 \%$ on the SSIM, the corresponding minimum SNR value to obtain meaningful information from the noisy PCE for each of $S_{\text {noisy } 1}$ and $S_{\text {noisy } 2}$ is $12.12 \mathrm{~dB}$ and $15.35 \mathrm{~dB}$, respectively, as presented in Fig. 13. Therefore, to improve the reconstructed results in Fig. 12, having an SNR of approximately $-6 \mathrm{~dB}$, the number of frame averages can be estimated to be 65 and 137, respectively, as shown in Fig. 14. The reconstructed results after frame-averaging are shown in Fig. 15.

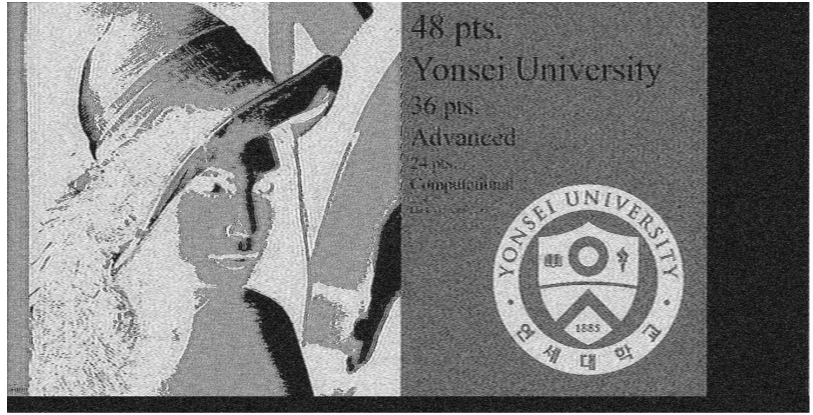

(a)

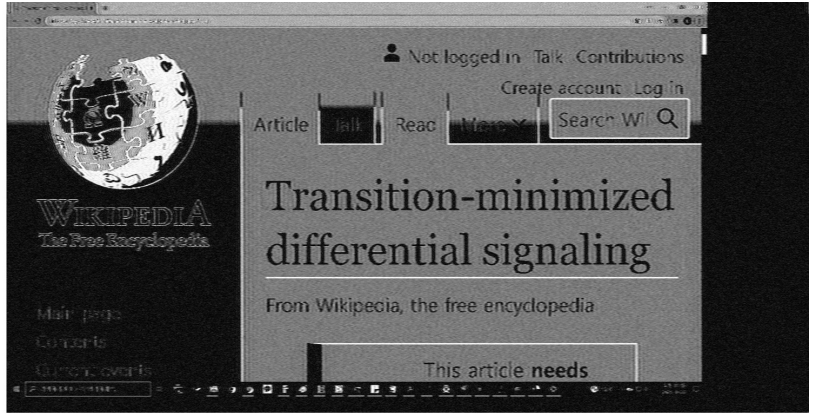

(b)

Figure 15. Reconstruct results using (a) 65 frames of synchronized $S_{\text {noisy } 1}$ and (b) 137 frames of synchronized $S_{\text {noisy2 }}$

As presented in Fig. 15, the reconstructed images after frame-averaging are more likely to provide useful information than the original images in Fig. 12. Furthermore, this analysis indicates that the SNR is not a unique parameter that represents the quality of the reconstructed image. Note that even though the SNRs of the received CE, including different information, are identical, some reconstructed images might not provide meaningful information.

Now, an analysis of the case where the previously defined $S_{f r 1}$ and $S_{f r 2}$ are collected in the SDR simultaneously, including additive noise, can be modeled, as in (19). The SNR from each perspective can be expressed as in (20).

$$
S_{m u l t}=\alpha \cdot S_{f r 1}+\beta \cdot S_{f r 2}+N_{A W G N} .
$$

$$
S N R_{1}[\mathrm{~dB}]=20 \cdot \log _{10} \frac{\alpha \cdot S_{f r 1(R M S)}}{\left(\beta \cdot S_{f r 2}+N_{A W G N}\right)_{(R M S)}} .
$$




$$
S N R_{2}[\mathrm{~dB}]=20 \cdot \log _{10} \frac{\beta \cdot S_{f r 2(R M S)}}{\left(\alpha \cdot S_{f r 1}+N_{A W G N}\right)_{(R M S)}} .
$$

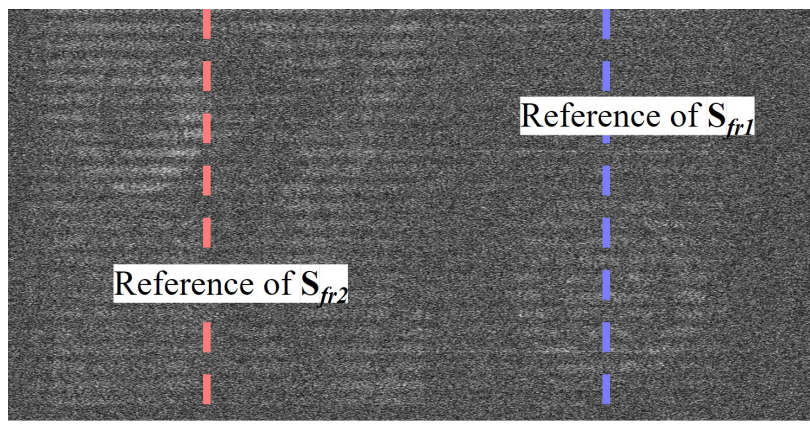

(a) $k=0$

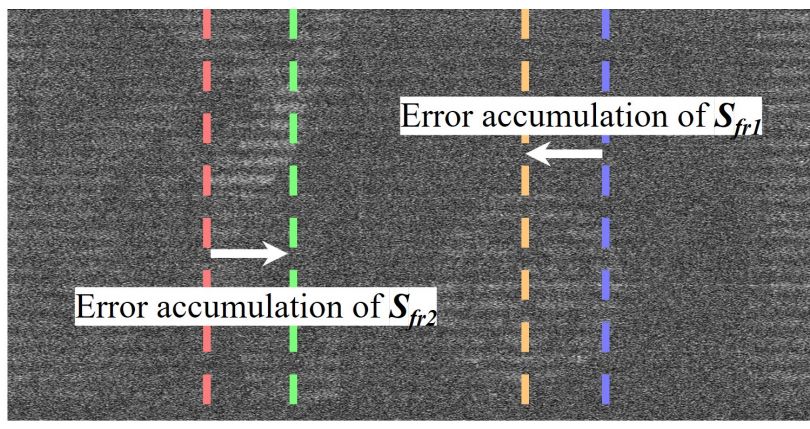

(b) $k=100$

Figure 16. Reconstruct results from (a) $u_{A M}\left[S_{m u l t . k=0}\right]$ and (b) $u_{A M}\left[S_{m u l t . k=100}\right]$

In (20), the parameters that use the subscript 1 are regarded as the $S_{\text {base }}$, as shown in Fig. 10-(a) and 2 for Fig. 10-(b). In addition, $P, f_{c}$, and the joint error parameters related to $\Delta$ and $\left(f_{c}-f_{L O}\right) / f_{L O}$ of $S_{f r 1}$ and $S_{f r 2}$ are the same as the previous assumption, and their scalars $\alpha$ and $\beta$ are 0.682 and 1, respectively, with $N_{A W G N}$. Under such conditions, the reconstructed information $u_{A M}\left[S_{m u l t}\right]$ at different $k$ values is shown in Fig. 16, and both $S N R_{1}$ and $S N R_{2}$ are set to $-10 \mathrm{~dB}$. Since the required minimum SNR to achieve meaningful information is $12.12 \mathrm{~dB}$ and $15.35 \mathrm{~dB}$ as discussed in the previous section, the frame average for $S_{m u l t}$ is conducted after the adjustment in (12). The expression for the frame average can be expressed as in (21).

$$
\begin{aligned}
& \left.S_{f a 1}[n, K]=\alpha \cdot S_{f r 1.0}[n]+\frac{1}{K}\left\{\sum_{k=0}^{K-1} \beta \cdot S_{f r 2 . k}[n] * \delta\left[n+k \Delta_{1}\right] \cdot e^{j 2 \pi k \Delta_{1}\left(f_{c}-f_{L O}\right)}+N_{A W G N}\right)\right\} . \\
& \left.S_{f a 2}[n, K]=\beta \cdot S_{f r 2.0}[n]+\frac{1}{K}\left\{\sum_{k=0}^{K-1} \alpha \cdot S_{f r 1 . k}[n] * \delta\left[n+k \Delta_{2}\right] \cdot e^{j 2 \pi k \Delta_{2}\left(f_{c}-f_{L O}\right)}+N_{A W G N}\right)\right\} .
\end{aligned}
$$

After frame-averaging, the $S_{f r}$ between the two remains, and the PCE of the opposite side, the coherency of which is not established, is eliminated by summation with $N_{A W G N}$ and division by $K$. Accordingly, the SNR variation as a function of the number of averaged frames $(K)$ is expressed in (22).

$$
\begin{aligned}
& S N R_{1}[K][d B]=20 \cdot \log _{10} \frac{\left(\alpha \cdot S_{f r 1.0}\right)_{R M S}}{\left(S_{f a 1}-\alpha \cdot S_{f r 1.0}\right)_{R M S}} . \\
& S N R_{2}[K][d B]=20 \cdot \log _{10} \frac{\left(\beta \cdot S_{f r 2.0}\right)_{R M S}}{\left(S_{f a 2}-\beta \cdot S_{f r 2.0}\right)_{R M S}} .
\end{aligned}
$$

As shown in Fig. 17, the minimum required number of the averaged frames are 154 and 354 for each case, and the reconstructed image of $u_{A M}\left\{S_{f a 1}[n, 154]\right\}$ and $u_{A M}\left\{S_{f a 2}[n, 354]\right\}$ are presented in Fig. 18.

As presented in Fig. 18, in contrast to the reconstruction from $S_{\text {noisy }}$, which includes only a single PCE, the reconstruction from $S_{\text {mult }}$ includes a wavy afterimage. The reason is that the unwanted PCE component is not perfectly eliminated in addition to the non-ideal effect of the demodulation process. Additional signal-processing techniques can effectively remove such noise [20].

Furthermore, an SNR-and SSIM-based analysis can be applied to counter the effects of poor electromagnetic propagation environments such as concrete or wooden walls existing in the line of sight between the target device under test (DUT) and antenna aperture. It can determine the duration of acquisition required to achieve a given SNR, provided that the electromagnetic characteristics are modeled, and the path loss of the obstacles is estimated [21], [22]. 


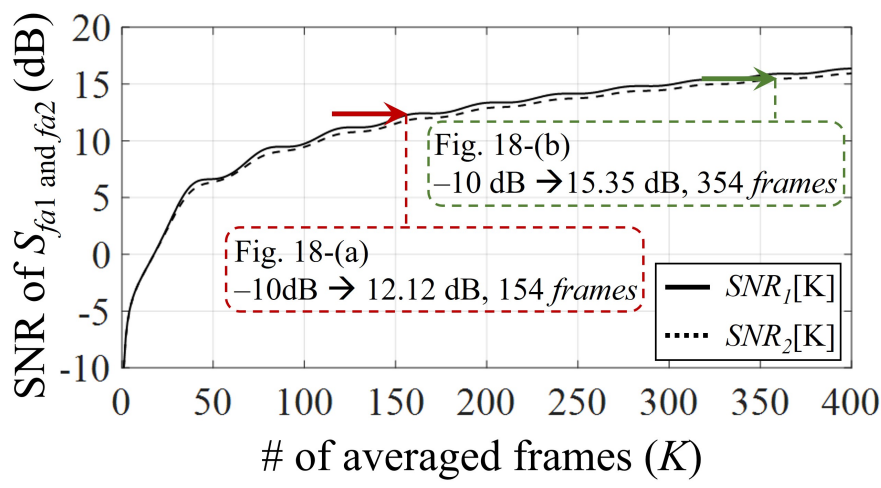

Figure 17. Increment of SNR as a function of averaged frame number $(K)$

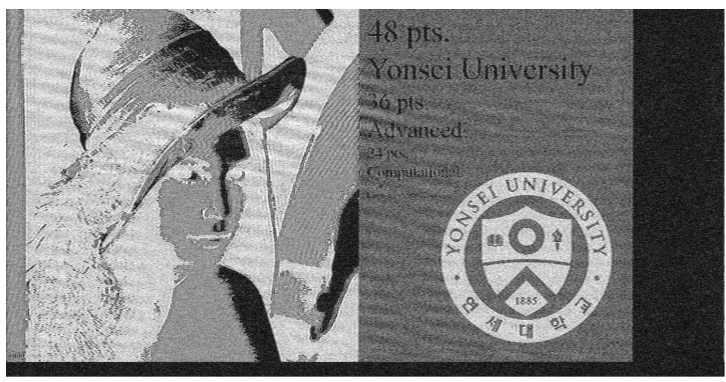

(a)

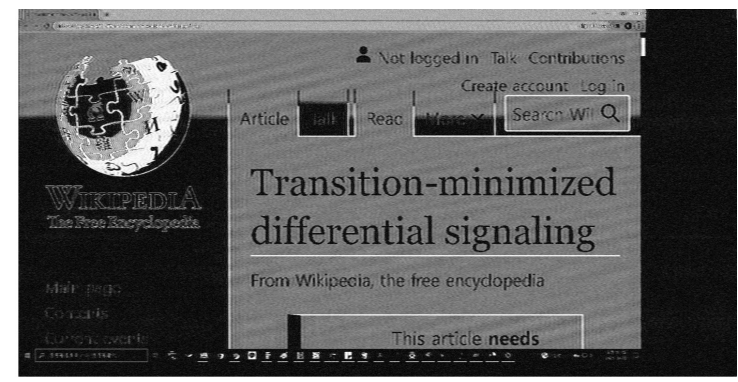

(b)

Figure 18. Reconstruct results from (a) $u_{A M}\left\{S_{f a 1}[n, 154]\right\}$ (b) $u_{A M}\left\{S_{f a 2}[n, 354]\right\}$

In this section, we describe the methodology for generating the PCE. In addition, we described a quantitative analysis of the possibility of information reconstruction based on the implemented PCE. Although this study handles a specific frequency band of $5 \%$ FBW centered at $(3+2 / 3) f_{p}$, the proposed methodology can be used to implement the PCE in other frequency bands as well. It is noteworthy that the applicability of the proposed analysis is not limited to environments with AWGN. It can be easily extended to environments containing various other types of noise, such as other communications as well as other types of EMI.

\section{Verification}

This section describes the reconstruction of the display information from the actual CE of the HDMI to validate the effectiveness of the proposed methodology. It provides comparisons between reconstructions from the implemented PCE, single frame (or single $k$ ), and multiple frames to be averaged from the actual CE. To acquire the actual CE from the HDMI, an experiment was conducted in the EMC chamber, as shown in Fig. 19.

The verification is conducted on $(3+2 / 3) f_{p}$, as in the previous analysis, and it utilizes an RF front-end bandwidth of $5 \%$.

\section{Reconstruction of actual-CE from single-VDU}

Figure 20 presents the reconstruction results from the PCE, single frame, and 84-frame average of the actual CE from the VDU presenting the target display information shown in Fig. 10-(a). The SNR of the received actual CE is estimated as $-4.8 \mathrm{~dB}$; frame-averaging improves the SNR to approximately $19.2 \mathrm{~dB}$, which provides a considerably higher amount of information.

Figure 21 presents the other reconstruction results from the PCE, single frame, and 140 averaged frames of the actual $\mathrm{CE}$, from the VDU presenting the target display information shown in Fig. 10-(b). The SNR of the received actual CE is estimated as $-5.6 \mathrm{~dB}$; frame-averaging improves the SNR by approximately $21.5 \mathrm{~dB}$, providing a considerably higher amount of information.

\section{Reconstruction of actual-CE from dual-VDU}

This section discusses the case in which two separate VDUs are placed at the same time and place. Fig. 21 shows the results for a single frame, targeting the information in Fig. 10-(a) with 88 averaged frames, as well as Fig. 10-(b) with 158 averaged frames. 

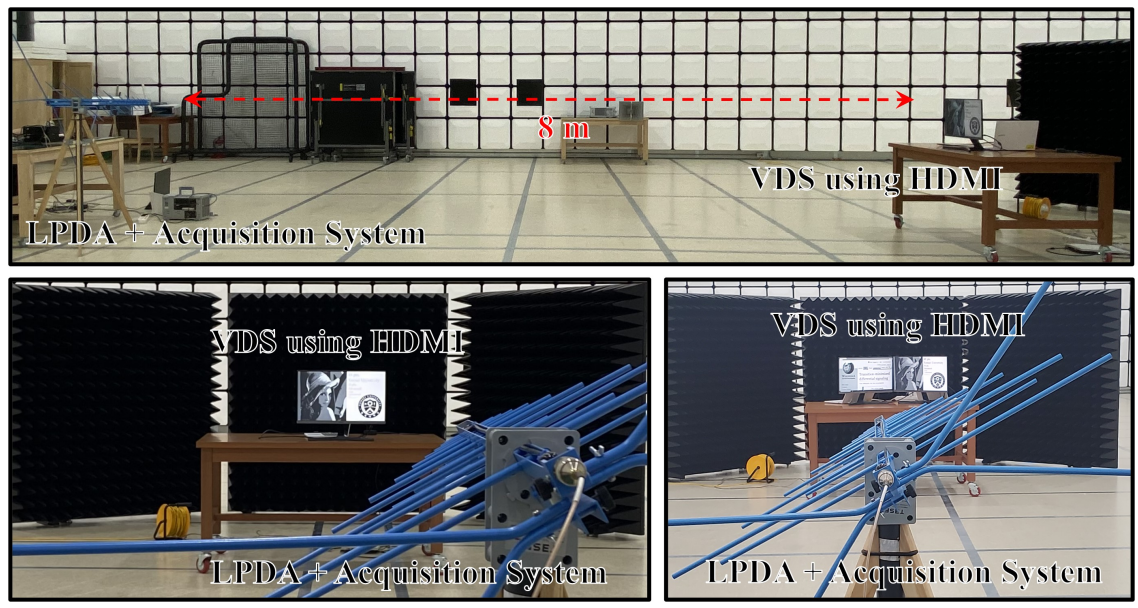

Figure 19. Measurement Set-up acquiring actual-CE

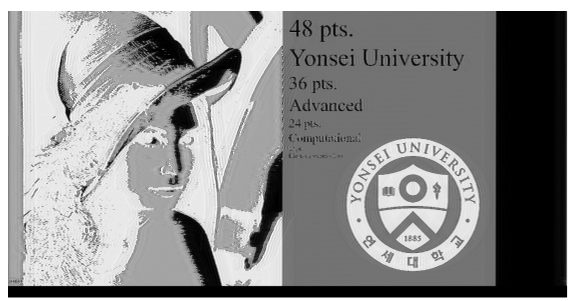

(a)

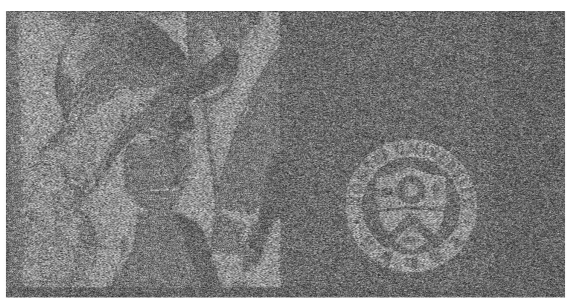

(b)

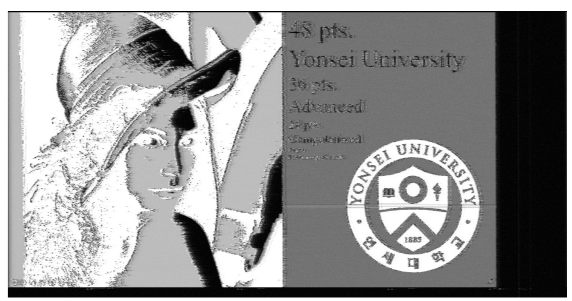

(c)

Figure 20. Result from (a) PCE, (b) single and (c) 84 averaged frames regarding the image shown in Fig. 10-(a)

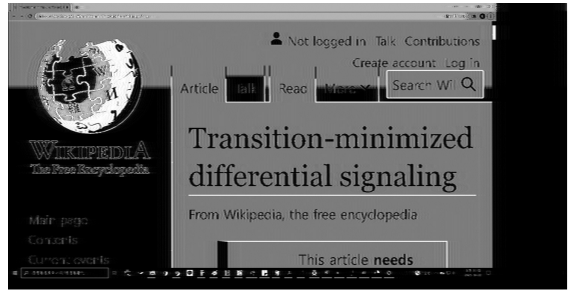

(a)

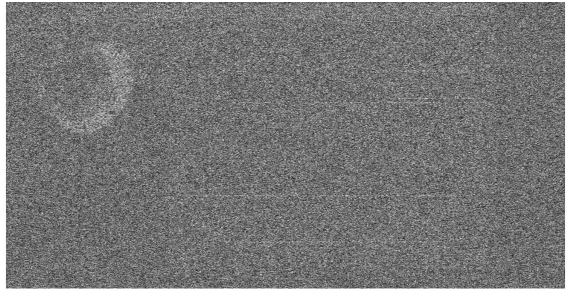

(b)

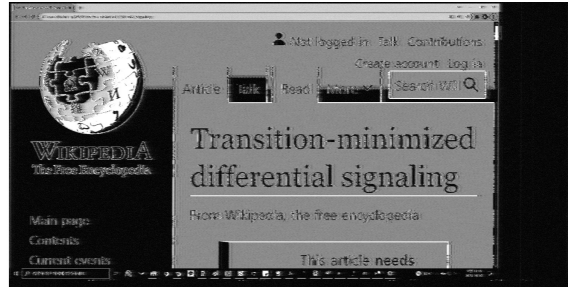

(c)

Figure 21. Result from (a) PCE, (b) single and (c) 140 averaged frames regarding the image shown in Fig. 10-(b)

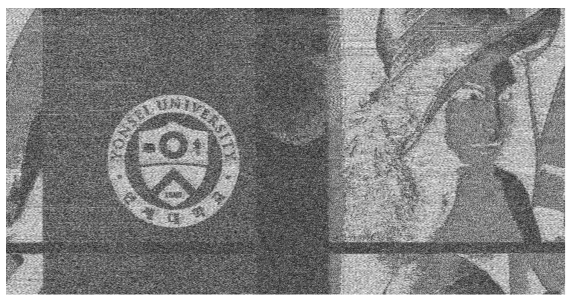

(a)

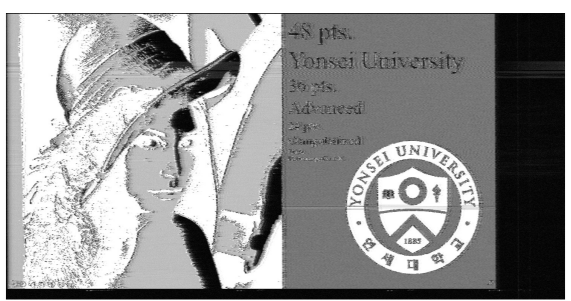

(b)

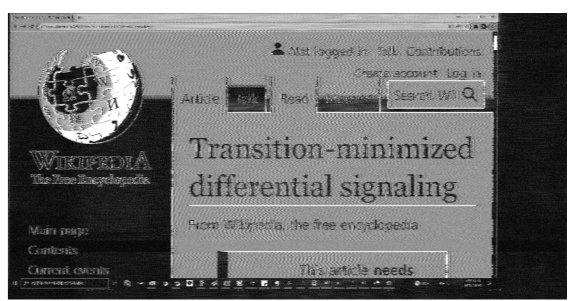

(c)

Figure 22. Result from (a) single frame,(b) 88 of averaged frames for Fig. 10-(a) and (c) 158 of averaged frames for Fig. $10-(b)$

Figure 22-(a) presents a reconstruction of the single frame from the simultaneous collection. In the case of Fig 22-(b), the signal strength of Fig. 10-(a) is higher than that of the other (Fig. 10-(b)); hence its reconstruction result is similar to the experiment conducted with a single VDU. However, as shown in Fig. 22-(c), the weaker one can be significantly affected and 
provides afterimages. These experimental results validate the effectiveness of our analysis and provide a quantitative index of how much acquisition is required in a realistic environment.

\section{Conclusion}

This paper proposes a quantitative analysis method on the possibility of information reconstruction by modeling EM leakage from HDMI among various SDIs currently used in public. First, to analyze the EM leakage characteristics of the HDMI, the signal flow in the cable is analyzed, and it is revealed that the baseband signal modulated in various forms is leaking in the form of multiple carrier frequencies which is related to the pixel update frequency $\left(f_{p}\right)$. Then, using these characteristics, modeled PCE can take into account the errors included in the VDU and the SDR. With the modeled PCE, the possibility of information reconstruction are quantified using an additional parameter named SSIM with SNR; besides, the improvement of SNR by the coherent frame-average as a function of the amount of averaged frames is presented. Finally, the validity of our proposed method is verified by comparing the results reconstructed from the actually measured CE from the HDMI. We hope this analytical methodology contributes to the area of information security.

\section{References}

1. G. McGraw, "Software security," in IEEE Security \& Privacy, vol. 2, no. 2, pp. 80-83, March-April 2004

2. K. Gandolfi, C. Mourtel, and F. Olivier, "Electromagnetic analysis: Concrete results," in Proc. Cryptographic Hardware Embedded Syst., 2001, pp. 251-261

3. Y. Hayashi et al., "Evaluation of information leakage from cryptographic hardware via common-mode current", IEICE Trans. Electron., vol. E95-C, no. 6, pp. 1089-1097, 2012

4. M. G. Kuhn, "Electromagnetic eavesdropping risks of flat-panel displays," in Proc. Privacy Enhancing Technologies, pp. 88-107, May 2005.

5. W. Van Eck, "Electromagnetic radiation from video display units: an eavesdropping risk?," Computers \& Security, vol. 4, pp. 269-286, 1985

6. M.G. Kuhn, "Compromising emanations: eavesdropping risks of computer displays", Technical Report UCAM-CL-TR-577, University of Cambridge, Computer Laboratory, Dec. 2003

7. M. Prvulovic, A. Zajić, R. L. Callan and C. J. Wang, "A Method for Finding Frequency-Modulated and Amplitude-Modulated Electromagnetic Emanations in Computer Systems," in IEEE Transactions on Electromagnetic Compatibility, vol. 59, no. 1, pp. 34-42, Feb. 2017

8. P. De Meulemeester, B. Scheers and G. A. E. Vandenbosch, "A Quantitative Approach to Eavesdrop Video Display Systems Exploiting Multiple Electromagnetic Leakage Channels," in IEEE Transactions on Electromagnetic Compatibility, vol. 62, no. 3, pp. 663-672, June 2020

9. L. Nowosielski, R. Przesmycki and M. Nowosielski, "Compromising Emanations from VGA and DVI Interface," in Proc. 2016 Progress in Electromagnetic Research Symposium (PIERS), 2016, pp. 1024-1028

10. T. Song, Y. Jeong and J. Yook, "Modeling of Leaked Digital Video Signal and Information Recovery Rate as a Function of SNR," in IEEE Transactions on Electromagnetic Compatibility, vol. 57, no. 2 pp. 164-172, April 2015

11. H. S. Lee, D. H. Choi, K. Sim and J. Yook, "Information Recovery Using Electromagnetic Emanations From Display Devices Under Realistic Environment," in IEEE Transactions on Electromagnetic Compatibility, vol. 61, no. 4, pp. 10981106, Aug. 2019

12. L. J. Cutrona, E. N. Leith, L. J. Porcello and W. E. Vivian, "On the application of coherent optical processing techniques to synthetic-aperture radar," in Proceedings of the IEEE, vol. 54, no. 8, pp. 1026-1032, Aug. 1966

13. Jian Li, Guoqing Liu and G. B. Giannakis, "Carrier frequency offset estimation for OFDM-based WLANs," in IEEE Signal Processing Letters, vol. 8, no. 3, pp. 80-82, March 2001

14. Video Electronics Standards Association (2013), VESA and Industry Standards and Guidelines for Computer Display Monitor Timing (DMT), Feb, 2013, https://glenwing.github.io/docs/VESA-DMT-1.13.pdf

15. P. De Meulemeester, B. Scheers and G. A. E. Vandenbosch, "Differential Signaling Compromises Video Information Security Through AM and FM Leakage Emissions," in IEEE Transactions on Electromagnetic Compatibility, vol. 62, no. 6, pp. 2376-2385, Dec. 2020

16. F. Elibol, U. Sarac and I. Erer, "Realistic eavesdropping attacks on computer displays with low-cost and mobile receiver system," in Proc. 2012 Proceedings of the 20th European Signal Processing Conference (EUSIPCO), 2012, pp. 1767-1771 
17. Y. Hayashi, N. Homma, M. Miura, T. Aoki, and H. Sone, "A threat for tablet PCS in public space: Remote visualization of screen images using EM emanation," in Proc. ACM SIGSAC Conf. Comput. Commun. Secur., 2014, pp. 954-965.

18. Y. Suzuki and Y. Akiyama, "Jamming technique to prevent information leakage caused by unintentional emissions of PC video signals," in Proc. IEEE Int. Symp. Electromagn. Compat., 2010, vol. 768, pp. 132-137.

19. Zhou Wang, A. C. Bovik, H. R. Sheikh and E. P. Simoncelli, "Image quality assessment: from error visibility to structural similarity," in IEEE Transactions on Image Processing, vol. 13, no. 4, pp. 600-612, April 2004.

20. X. Liu, S. Wang, S. Fu, L. Yuliang, S. Liu and W. Zhou, "An Efficient Collaborative Filtering Method for Image Noise and Artifact Removal," in IEEE Access, vol. 8, pp. 124158-124171, 2020

21. G. Daian, A. Taube, A. Birnboim, et al., "Modeling the dielectric properties of wood", Wood Sci Technol 40, pp. 237-246, 2006

22. K. Cho, S. Jo, Y. H. Noh, N. Lee, S Kim and J Yook, "Complex Permittivity Measurements of Steel Fiber-Reinforced Cementitious Composites Using a Free-Space Reflection Method with a Focused Beam Lens Horn Antenna", Sensors. 2021, 21(23):7789.

\section{Acknowledgments}

This work was supported by support for standardization and certification of new energy technologies of the Korea Institute of Energy Technology Evaluation and Planning (KETEP) grant funded by the Korea government Ministry of Trade, Industry and Energy. (No. 20217301010050)

\section{Author contributions}

E. Lee conceived the idea, designed the structure, and wrote the main ideas. E. Lee, D. Choi, and T. Nam conducted the experiments together. J. Yook revised the manuscript, contributed some ideas, and supervised the work.

\section{Competing interests}

The authors declare no competing interests.

\section{Data availability}

The methodology of generation and analysis of all data during this study are included in this published article. 